\title{
CANCER \\ A new mouse model of melanoma progression
}

Sun, Q. et al. Nat. Commun. 10, 5023 (2019)

Melanoma, the most serious form of skin cancer, is easier to treat when detected early. Efforts are therefore underway to develop strategies for early detection and intervention, but these have been hampered by a lack of animal models recapitulating human disease. Using a novel mouse model, a study published in Nature Communications provides new insights into melanoma progression, thereby opening new avenues for the development of diagnostic and therapeutic tools.

The mouse is the most widely used preclinical model for melanomagenesis. However, in most murine models melanoma arises in the dermis, whereas human melanoma initiates from skin epidermis. This dermal discrepancy calls into question the validity of these models in studying the early stages of melanoma. In addition, the melanoma field lacks CreER lines that target specific melanocyte populations, limiting our understanding of the cellular origin of melanoma. "There is recent controversy about whether melanocyte stem cells (McSCs) in the hair follicles are the cellof-origin of melanoma," explains Qi Sun, first author on the paper and postdoctoral fellow in the lab of Mayumi Ito at New York University School of Medicine.

In 2017, a team of investigators from Cornell University, Ithaca, USA demonstrated that hair follicle McSCs do have the potential to form tumors (Moon et al). A team from VIB Center for Cancer Biology in Leuven, Belgium suggested otherwise. Their results showed that McSCs cannot form melanoma, and they identified mature, melanin-producing melanocytes as the cancer-initiating cells (Kohler et al). In both studies, the investigators used the same well-known Tyr-CreER:Braf:Pten genetically modified mouse model of melanoma, in which CreER recombinase expression is under the control of a melanocyte-specific tyrosinase (Tyr) gene promoter. In these mice, tamoxifen treatment induces Cre-lox recombination specifically in melanocytes, resulting in Braf ${ }^{V 600 E}$ expression, Pten tumor suppressor gene silencing, and subsequent

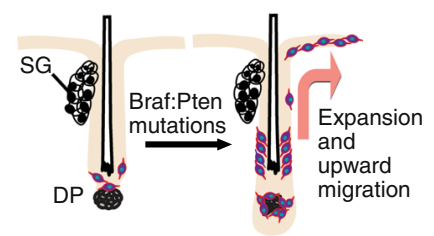

Schematic model showing that upon oncogenic mutations, melanocyte stem cells expand in the niche and migrate upward to the epidermis. SG, sebaceous gland, DP dermal papilla. Reprinted with permission from Sun et al. (2019)

Springer Nature.

development of melanoma. However, in this model, melanocytes can be affected in different places in the mouse. Several studies have reported that Tyr-CreER targets both McSCs located in the hair follicle and melanocytes in the dermis, which might account for the conflicting results observed in the two studies.

"We sought to establish a novel mouse melanoma model that would enable us to address the controversy of whether McSC is a cellular origin of melanoma and that would better mimic human melanoma initiation and progression," says Sun. Using a $c$-kit promoter that specifically targets follicular McSCs while leaving dermal melanocytes be, they generated mice that express Braf ${ }^{V 600 E}$ and delete Pten only in McSCs upon tamoxifen induction. Analysis of the c-Kit-CreER:Braf:Pten mice 10 days after initial tamoxifen treatment showed that induction of oncogenic mutations in McSCs induced their ectopic proliferation and upward migration into the upper follicular compartment and the epidermis. Contrary to Tyr-CreER:Braf:Pten mice that showed simultaneous expansion of epidermal and dermal melanoma cells at the same time point, melanoma initiation in c-Kit-CreER:Braf:Pten mice was confined to the epithelial compartment, mimicking the initial radial growth phase of human melanoma. Analysis at a later time point (26 days after tamoxifen treatment) revealed that epidermal c-Kit-CreER:Braf:Pten McSCderived oncogenic melanocytes had invaded the dermis, similarly to the vertical growth phase of human melanoma progression.

Sun and colleagues also demonstrated that a second hit such as UVB irradiation-a carcinogen agent commonly used to enhance the development of melanomawas not necessary to induce McSC transformation. "We showed that normal regenerative signals Wnt and endothelin, provided by epithelial niche cells, are sufficient to drive the formation of epidermal melanoma from oncogenic McSCs. The new c-Kit melanoma model may be better than previous models in studying the initiation and early progression of human melanoma," explains Sun.

Next, the investigators compared the transcriptional profiles of murine McSCs and their derived melanoma. McSC transformation involved downregulation of melanocytic genes and upregulation of extracellular matrix components, melanoma markers and neuronal and/or neural crest genes-all changes reminiscent of those observed in human melanoma. Single-cell sequencing also revealed that McSC-derived murine melanoma were transcriptionally heterogeneous, another hallmark of human disease.

"We plan to use this new tool to better understand the molecular mechanisms regulating each step of melanoma initiation and progression," says Sun, adding that their new mouse model might become invaluable to screen future therapies. "New immunotherapeutic possibilities are frequently proposed and many are rushed to clinical trials, yet these take years to generate results and can only test a few variables at one time. We propose that this new model provides such a unique opportunity to test new therapies and their potential associated risks," concludes Sun.

Alexandra Le Bras

Published online: 16 December 2019 https://doi.org/10.1038/s41684-019-0447-y 